\title{
Direct in vivo measurement of glycine and the neurochemical profile in the rat medulla oblongata
}

\author{
Lijing Xin $^{\mathrm{a} *}$, Giulio Gambarota ${ }^{a}$, João M. N. Duarte ${ }^{\mathrm{b}}$, Vladimír Mlynárik ${ }^{\mathrm{a}}$ \\ and Rolf Gruetter ${ }^{a, b, c}$
}

\begin{abstract}
The medulla oblongata (MO) contains a high density of glycinergic synapses and a particularly high concentration of glycine. The aims of this study were to measure directly in vivo the neurochemical profile, including glycine, in MO using a spin-echo-based ${ }^{1} \mathrm{H}$ MRS sequence at TE $=\mathbf{2 . 8} \mathrm{ms}$ and to compare it with three other brain regions (cortex, striatum and hippocampus) in the rat. Glycine was quantified in $\mathrm{MO}$ at $\mathrm{TE}=\mathbf{2 . 8} \mathrm{ms}$ with a Cramér-Rao lower bound (CRLB) of approximately $5 \%$. As a result of the relatively low level of glycine in the other three regions, the measurement of glycine was performed at TE $=20 \mathrm{~ms}$, which provides a favorable J-modulation of overlapping myo-inositol resonance. The other 14 metabolites composing the neurochemical profile were quantified in vivo in MO with CRLBs below 25\%. Absolute concentrations of metabolites in MO, such as glutamate, glutamine, $\gamma$-aminobutyrate, taurine and glycine, were in the range of previous in vitro quantifications in tissue extracts. Compared with the other regions, MO had a three-fold higher glycine concentration, and was characterised by reduced $(p<0.001)$ concentrations of glutamate $(-50 \pm 4 \%)$, glutamine $(-54 \pm 3 \%)$ and taurine $(-78 \pm 3 \%)$. This study suggests that the functional specialisation of distinct brain regions is reflected in the neurochemical profile. Copyright (C) 2010 John Wiley \& Sons, Ltd.
\end{abstract}

Keywords: glycine; neurochemical profile; medulla oblongata; NMR spectroscopy

\section{INTRODUCTION}

The medulla oblongata (MO) is a part of the brainstem that regulates motor and sensory functions, as well as autonomic processes, such as heart beat, breathing and blood pressure. In MO, glycine (Gly) is present at a particularly high concentration. Gly has a dual role as an inhibitory neurotransmitter, activating Gly receptors, and as a co-agonist for excitatory $N$-methyl-Daspartate receptors (1). In addition to its roles in neurotransmission and neuromodulation, Gly is the precursor of metabolites such as purines, creatine $(\mathrm{Cr})$ and glutathione (GSH), and may be incorporated in lipids and proteins (2). The enhancement of Gly release in the brainstem has been proposed to be a neuroprotective mechanism against metabolic noxious stimuli, such as hypoxia, hypoglycaemia and ischaemia (3). In addition, lesions of $\mathrm{MO}$ are involved in several pathologies, such as hypertension (4) and multiple sclerosis (5). Therefore, it is of interest to investigate in vivo MO, with a specific focus on Gly tissue levels.

Localised MRS, as a noninvasive technique of exploring neurochemical information $(6,7)$, is a desirable choice for the in vivo measurement of the neurochemical profile, including Gly in MO. As Gly is present at low concentrations in the forebrain and its resonance signal overlaps with the larger resonance of myo-inositol (Ins) in the ${ }^{1} \mathrm{H}$ MR spectrum, in vivo detection of Gly by MRS is challenging. In a number of studies, Gly tissue levels have been measured in human brain $(8,9)$. Recently, the direct detection of Gly in rat brain in vivo has been reported, using spin-echo excitation at TE $=20 \mathrm{~ms}$ (10). To our knowledge, in vivo
* Correspondence to: L. Xin, Lijing Xin, Ecole Polytechnique Fédérale de Lausanne (EPFL), SB-IPSB-LIFMET, CH F1 633, Station 6, CH-1015 Lausanne, Switzerland.

E-mail: lijing.xin@epfl.ch

a L. Xin, G. Gambarota, V. Mlynárik, R. Gruetter

Laboratory of Functional and Metabolic Imaging (LIFMET), Ecole Polytechnique Fédérale de Lausanne, Lausanne, Switzerland

b J. M. N. Duarte, R. Gruetter

Department of Radiology, University of Lausanne, Lausanne, Switzerland

c R. Gruetter

Department of Radiology, University of Geneva, Geneva, Switzerland

Contract/grant sponsors: Centre d'Imagerie BioMédicale (CIBM) of University of Lausanne (UNIL), University of Geneva (UNIGE), Hôpitaux Universitaires de Genève (HUG), Centre Hospitalier Universitaire Vaudois (CHUV), Ecole Polytechnique Federale de Lausanne (EPFL); Leenaards and Jeantet Foundations.

Abbreviations used: Ala, alanine; ANOVA, analysis of variance; Asc, ascorbate; Asp, aspartate; $B H B, \beta$-hydroxybutyrate; $C O$, cortex; $C r$, creatine; $C R L B$, Cramér-Rao lower bound; FAST(EST)MAP, fast, automatic shim technique using echo-planar signal readout for mapping along projections; FID, free induction decay; GABA, $\gamma$-aminobutyrate; Glc, glucose; Gln, glutamine; Glu, glutamate; Gly, glycine; GPC, glycerophosphocholine; GSH, glutathione; HI, hippocampus; Ins, myo-inositol; Lac, lactate; MO, medulla oblongata; NAA, $\mathrm{N}$-acetylaspartate; NAAG, N-acetylaspartylglutamate; NT, number of transients; $P C h o$, phosphocholine; $P C r$, phosphocreatine; $P E$, phosphoethanolamine; Scyllo, scyllo-inositol; SEM, standard error of the mean; SNR, signal-to-noise ratio; SPECIAL, spin-echo, full-intensity acquired localised; ST, striatum; Tau, taurine; $t C r$, total creatine; VAPOR, variable power radiofrequency pulses with optimised relaxation delays; VOI, volume of interest. 
measurement of Gly and the neurochemical profile in the rat $\mathrm{MO}$ has not been reported to date.

In this study, we hypothesised that the combination of short-TE spin-echo excitation localisation and high magnetic field strength $(9.4 \mathrm{~T})$ would provide a high spectral resolution and signalto-noise ratio (SNR), which would, in turn, allow a thorough investigation of the neurochemical profile of the medulla. Therefore, the aims of this investigation were to measure in vivo the neurochemical profile of rat $M O$ by localised ${ }^{1} \mathrm{H}$ NMR spectroscopy, with particular focus on Gly quantification at $\mathrm{TE}=2.8 \mathrm{~ms}$, and to compare the neurochemical profile of $\mathrm{MO}$ with that of the hippocampus (HI), striatum (ST) and cortex (CO).

\section{METHODS}

\section{Animal preparation}

Experiments were performed on Sprague Dawley rats $(274 \pm 16 \mathrm{~g}, n=6)$ under isoflurane anaesthesia $(1.5-2 \%$ in oxygen). The animals were placed in a homebuilt holder, and the head was fixed by a bite bar and a pair of ear bars. The respiration rate was monitored by a small animal monitor (SA Instruments Inc., Stony Brook, NY, USA) and the body temperature was measured by a rectal thermosensor and maintained at $38.0 \pm 0.5^{\circ} \mathrm{C}$ by circulating heated water. All animal procedures were performed according to federal guidelines and were approved by the local ethics committee.

\section{MRS}

Proton NMR experiments were performed on a Varian INOVA console (Varian, Palo Alto, CA, USA) interfaced to an actively shielded $9.4 \mathrm{~T} / 31 \mathrm{~cm}$ magnet (Magnex Scientific, Abingdon, Oxfordshire, UK) with a $12 \mathrm{~cm}$ inner diameter, actively shielded gradient $(400 \mathrm{mT} / \mathrm{m}$ in $120 \mu \mathrm{s})$. A homebuilt geometrically decoupled ${ }^{1} \mathrm{H}$ quadrature surface coil $(17 \mathrm{~mm}$ in diameter) was used as transceiver. Fast automatic shimming employing the fast, automatic shim technique using echo-planar signal readout for mapping along projections [FAST(EST)MAP] (11) was used to adjust all first- and second-order shims.

In vivo proton spectra were acquired using the spin-echo, full-intensity acquired localised (SPECIAL) spectroscopy method, which consists of a $2 \mathrm{~ms}$ slice-selective full-passage adiabatic pulse (hyperbolic secant 10) with a bandwidth of $10 \mathrm{kHz}\left(\gamma \mathrm{B}_{1} /\right.$ $2 \pi=3.7 \mathrm{kHz}$ ) applied on alternate scans, followed by a spin-echo sequence $\left[0.5 \mathrm{~ms} / 90^{\circ}\right.$ and $1 \mathrm{~ms} / 180^{\circ}$ asymmetric slice-selective pulses with bandwidths of $13.5 \mathrm{kHz}, \gamma \mathrm{B}_{1} / 2 \pi=3.35 \mathrm{kHz}$ and $6.2 \mathrm{kHz}, \gamma \mathrm{B}_{1} / 2 \pi=4.95 \mathrm{kHz}$, respectively (12)]. Two TEs, i.e. 2.8 and $20 \mathrm{~ms}$, were used for the assessment of the neurochemical profile in all four cerebral regions and for the Gly measurement in $\mathrm{CO}, \mathrm{HI}$ and ST, respectively. Water suppression was achieved using variable power radiofrequency pulses with optimised relaxation delays (VAPOR) (13) and three outer volume saturation modules were interleaved with water suppression elements (13). Volumes of interest (VOIs) of $4 \times 2 \times 4,6 \times 1.5 \times 2,3 \times 2.5 \times 2$ and $3 \times 2 \times 2 \mathrm{~mm}^{3}$ were located in $\mathrm{MO}, \mathrm{CO}, \mathrm{ST}$ and $\mathrm{HI}$, respectively. The surface coil was shifted to the posterior part of the head for the measurement of MO. Images of rat brain acquired with a fast spin-echo sequence (matrix, $128 \times 128 ; \mathrm{TE}=40 \mathrm{~ms}$; echo train length, $8 ; \mathrm{TR}=4 \mathrm{~s}$; slice thickness, $1 \mathrm{~mm} ; 13$ slices; 4 averages) were used for the positioning of the VOls. Flip angles in SPECIAL were adjusted for each VOI. All spectra were acquired with the following acquisition parameters: spectral width, $5 \mathrm{kHz} ; 4096$ complex data points; $\mathrm{TR}=4 \mathrm{~s}$. The acquisition at each TE consisted of an array of free induction decays (FIDs) (16 averages for each FID) that were saved separately [CO: number of transients (NT), 240; HI: NT=240-480; ST: NT=480-640; MO: $\mathrm{NT}=800-960)$. Spectra acquired without water suppression at the corresponding TE were used as internal reference for the quantification of metabolite concentrations. $B_{0}$ shift correction was performed prior to summation of the spectra, and eddy current effects were corrected with the acquired reference water signal.

\section{Data analysis}

Spectral data analysis was performed with LCModel (14), using a basis set containing simulated metabolite spectra and a macromolecular baseline measured by the inversion recovery method (15). As a result of the use of short TE, the effect of an inhomogeneous $B_{1}$ within the voxel (approximately 15-30\% variation) on the evolution of J-coupled resonances, reported by Snyder et al. (16), was considered to be negligible. Therefore, nominal flip angles were used in the simulation of the spectra. For the quantification of short-TE spectra $(T E=2.8 \mathrm{~ms}$ ), the unsuppressed water signal was used as an internal reference. Regional water content differences were taken into account when assessing the metabolite quantification (17). The Gly concentration in $\mathrm{CO}, \mathrm{HI}$ and $\mathrm{ST}$ was obtained from spectra acquired at $\mathrm{TE}=20 \mathrm{~ms}$ using the regional total creatine $(\mathrm{tCr})$ concentration obtained at $\mathrm{TE}=2.8 \mathrm{~ms}$ as the internal reference, assuming similar $\mathrm{T}_{2}$ relaxation at $9.4 \mathrm{~T}$ (18). The spectral basis sets included the following metabolites: alanine (Ala); lactate (Lac); $\gamma$-aminobutyrate (GABA); $N$-acetylaspartate (NAA); glutamate (Glu); glutamine (GIn); aspartate (Asp); $\mathrm{Cr}$; phosphocreatine (PCr); glycerophosphocholine (GPC); taurine (Tau); Ins; Gly; GSH; phosphocholine (PCho); ascorbate (Asc); scyllo-inositol (Scyllo); $\mathrm{N}$-acetylaspartylglutamate (NAAG); $\beta$-hydroxybutyrate (BHB); glucose (Glc); phosphoethanolamine (PE). All results were presented as the mean \pm standard error of the mean (SEM), and were compared with two-way analysis of variance (ANOVA) followed by Bonferroni's multiple comparison test.

\section{RESULTS}

With the ${ }^{1} \mathrm{H}$ quadrature surface coil (diameter, $17 \mathrm{~mm}$ ), images acquired with the fast spin-echo sequence showed sufficient coverage of the rat brain, even the ventral part (Fig. 1), to allow volume positioning in MO. The typical linewidth of the water resonance was $10-12 \mathrm{~Hz}$ for $\mathrm{CO}, \mathrm{HI}$ and $\mathrm{ST}$, and $15-17 \mathrm{~Hz}$ for $\mathrm{MO}$. In vivo proton $\mathrm{MR}$ spectra of $\mathrm{MO}$ in rat brain were obtained with an SNR (measured for the NAA singlet before applying apodisation, 800-960 averages) of $16 \pm 2(n=6)$. Compared with the spectra of $\mathrm{CO}, \mathrm{ST}$ and $\mathrm{HI}$, the spectrum of MO showed a significant reduction in Tau resonances at 3.42 and $3.25 \mathrm{ppm}$, as well as of Glu and Gln resonances (Fig. 1). In addition, the peak of Ins at approximately $3.55 \mathrm{ppm}$ was unusually high. Using the basis set that did not contain the Gly resonance led to a substantial fitted residual and slight distortion of baseline at $3.55 \mathrm{ppm}$ in the LCModel analysis of in vivo NMR spectra obtained from MO (Fig. 2), which indicates the contribution of Gly with a resonance peak at $3.55 \mathrm{ppm}$. Adding the Gly spectrum to the basis set, this fitted residual was reduced to noise level and the baseline became smooth. The concentration of Gly was 


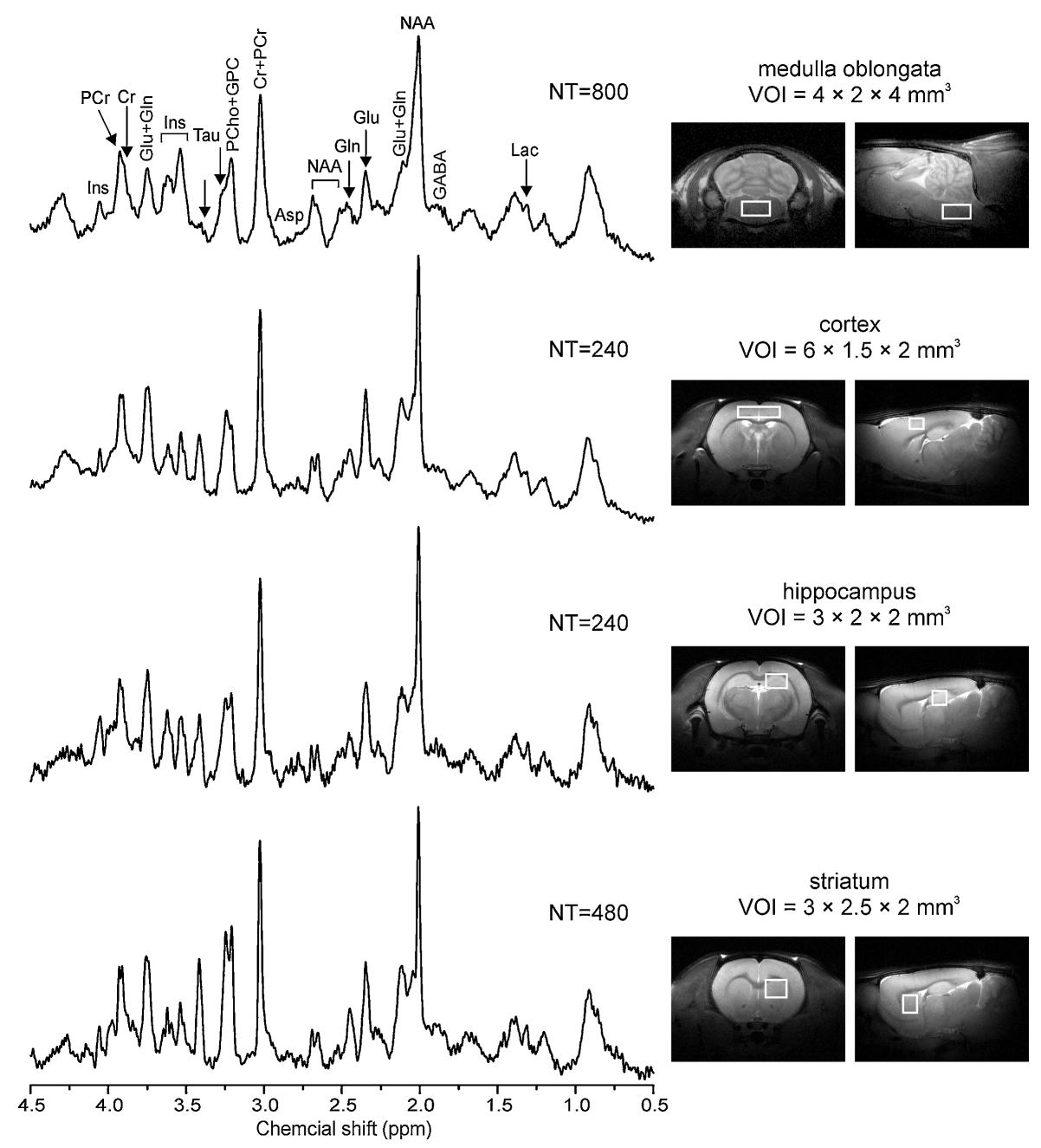

Figure 1. Representative in vivo ${ }^{1} \mathrm{H}$ NMR spectra acquired with the spin-echo, full-intensity acquired localised (SPECIAL) sequence at $9.4 \mathrm{~T}$ from the volumes of interest (VOls) located in the medulla oblongata, cortex, hippocampus and striatum $[\mathrm{TE}=2.8 \mathrm{~ms}$, TR=4 $\mathrm{s}$, number of transients $(N T)=240-800]$. Fourier transform and Gaussian function weighting $\left(\exp \left[-(t-0.03)^{2} / 0.12^{2}\right]\right)$ were applied. No baseline correction or water signal removal was applied. See text for abbreviations.

quantified at $2.9 \pm 0.2 \mu \mathrm{mol} / \mathrm{g}(n=6)$ with CRLB of $5.3 \pm 0.1 \%$ (Fig. 3a).

A neurochemical profile of 15 metabolites including Asp, $\mathrm{Cr}$, PCr, GABA, Gln, Glu, Ins, Lac, NAA, Tau, Glc, NAAG, PE, Gly and $\mathrm{GPC}+\mathrm{PCho}$, was quantified in $\mathrm{MO}$ at $\mathrm{TE}=2.8 \mathrm{~ms}$ with $\mathrm{CRLBs}$ lower than $25 \%$ (Fig. 3b). In addition, Gly was measured in $\mathrm{CO}, \mathrm{HI}$ and $\mathrm{ST}$ at $\mathrm{TE}=20 \mathrm{~ms}$, with CRLBs below 25\%, and its concentration was $0.92 \pm 0.04 \mu \mathrm{mol} / \mathrm{g} \quad(n=6), \quad 1.04 \pm 0.15$ $\mu \mathrm{mol} / \mathrm{g}(n=5)$ and $0.95 \pm 0.08 \mu \mathrm{mol} / \mathrm{g}(n=6)$, respectively. These values were, on average, three-fold lower than that in $\mathrm{MO}$ $(p<0.001, n=6)$ (Fig. 3a). Other marked differences were noted in the neurochemical profile of MO compared with the other three brain regions: significantly lower $(p<0.001, n=6)$ concentrations of Glu $(-50 \pm 4 \%), \operatorname{Gln}(-54 \pm 3 \%)$ and Tau $(-78 \pm 3 \%)$ as averaged from $\mathrm{CO}, \mathrm{HI}$ and $\mathrm{ST}$, respectively. Concentrations of $\mathrm{PE}$ were lower in $\mathrm{MO}$, reaching half of the concentration observed in $\mathrm{HI}(-49 \pm 7 \%, p<0.01, n=6)$ and ST $(-53 \pm 6 \%, p<0.01, n=6)$. The levels of $\mathrm{Cr}$ in $\mathrm{MO}$ tended to be lower than in the other regions. For instance, compared with $\mathrm{HI}$, the concentration of $\mathrm{Cr}$ in $\mathrm{MO}$ was $42 \pm 2 \%$ lower $(p<0.001$, $n=6)$. The concentration of NAA in MO was similar to that in ST, but lower than that in $\mathrm{CO}(-24 \pm 4 \%, p<0.001, n=6)$ and $\mathrm{HI}$ $(-27 \pm 4 \%, p<0.001, n=6)$.

\section{DISCUSSION}

This study demonstrated the feasibility of using in vivo ${ }^{1} \mathrm{H}$ NMR spectroscopy to determine the neurochemical profile in rat MO, composed of 15 metabolites including Gly, the content of which may reflect the high density of glycinergic neurons (19). The low CRLBs of these metabolites (below 25\%) indicate the high reliability of the current measurement. This can be ascribed to the high SNR and spectral resolution at $9.4 \mathrm{~T}$, the use of a large quadrature surface coil that covers the ventral part of the rat brain, and the sensitivity improvement using an advanced localised spectroscopic technique at short TE. Water suppression and outer volume suppression sufficiently suppressed unwanted signals, such as lipid signals and large water signals, which were not observed in the spectra of MO despite the location at the brainstem (Fig. 1). An excellent agreement of the concentrations of Glu, Gln, 


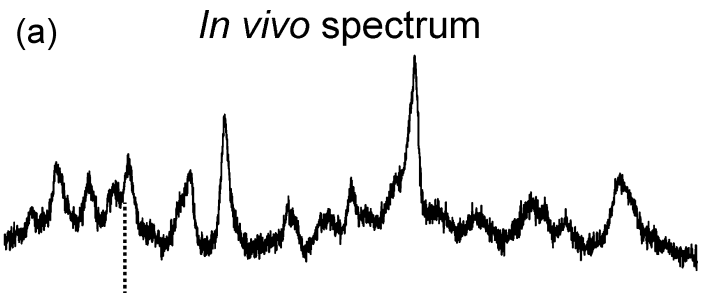

basis set w/o Gly
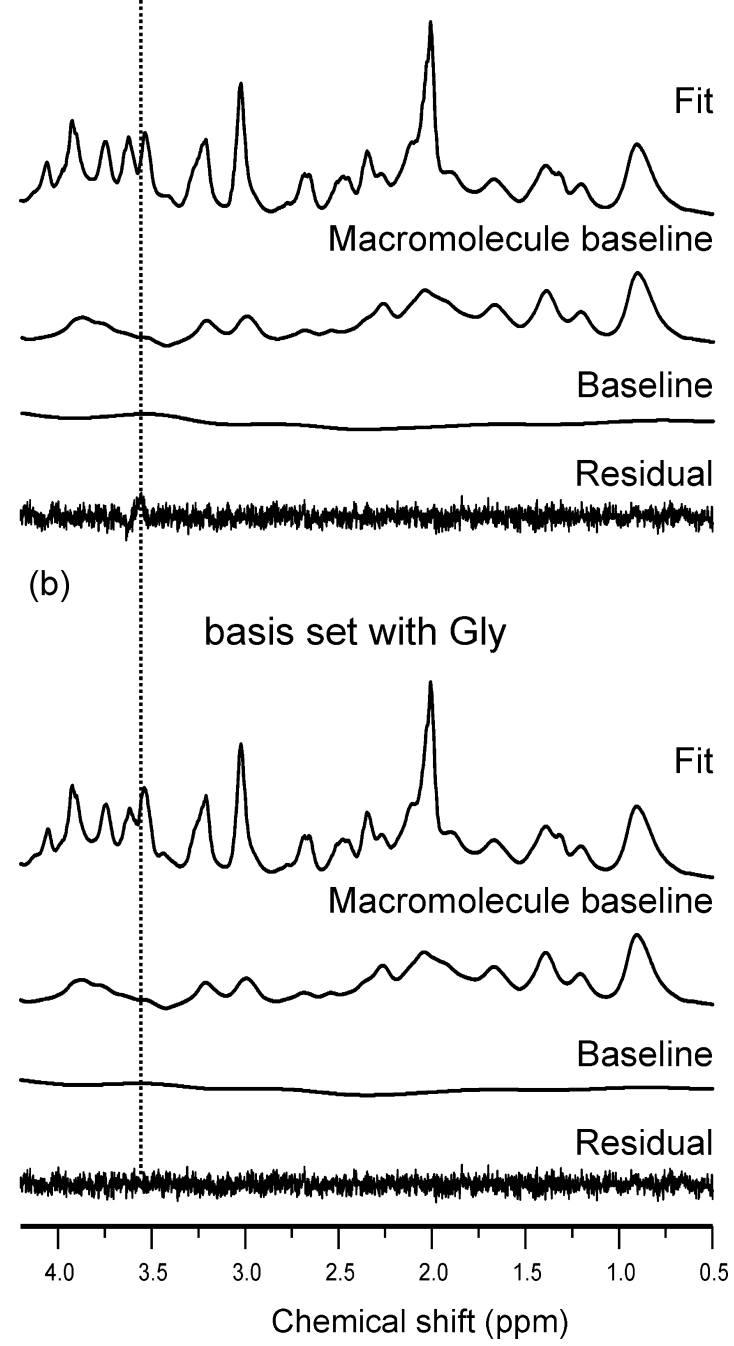

Figure 2. LCModel analysis of in vivo spectrum of the rat medulla oblongata (data from the same rat as shown in Fig. 1, no apodisation was applied) using the basis set without (a) and with (b) glycine (Gly). The corresponding fit, macromolecule baseline, baseline and residual are shown.

GABA and Tau in MO was found with previously quantified values in tissue extracts (20).

The measurement in $\mathrm{MO}$ using the surface coil was more challenging than that in other brain regions because of its deep location in the posterior part of the brain. However, the SNR obtained was still sufficient for the quantification of 15 metabolites of the neurochemical profile, which can be ascribed to the use of the short TE and high magnetic field. After first- and second-order shimming adjustment, the linewidth of the water signal was $15-17 \mathrm{~Hz}$ in $\mathrm{MO}$, which was approximately $5 \mathrm{~Hz}$ greater than that in the other regions; this was attributed mainly to the increased magnetic field inhomogeneity because of susceptibility effects induced by the nearby sinuses.

Gly was also measured in vivo in $\mathrm{MO}$ at $\mathrm{TE}=2.8 \mathrm{~ms}$ with a high precision, as judged from the low CRLB of $5 \%$ (Fig. 3). The concentration of Gly in MO measured in this study is in the range of previous reports using biochemical assays (20), and is somewhat lower than the value published by Aprison et al. (21), possibly as a result of the age difference of the rats (22).

Recently, threonine, which has one doublet close to the Gly resonance peak at $3.55 \mathrm{ppm}$, was quantified in vivo in rat brain (23) and human brain (24). Additional LCModel analysis using the basis set including threonine was performed to investigate the effect on the assessment of Gly (data not shown). However, the resonance peaks of threonine and Lac at $1.32 \mathrm{ppm}$ are undistinguishable for LCModel, which tends to assign the whole peak to threonine, leading to strong overestimation of threonine and underestimation of Lac concentration. Consequently, the Gly concentration would also be systematically underestimated. The overestimated concentration of threonine was between 1.1 and $2.6 \mathrm{~mm}$, which leads to a 9-21\% alteration in Gly level. As the concentration of threonine should be $0.6 \pm 0.2 \mathrm{~mm}(23)$, its effect on the quantification of Gly is expected to be less than $9 \%$.

In CO, ST and HI, Gly cannot be reliably quantified at $\mathrm{TE}=2.8$ ms because of its lower regional concentration (approximately $1 \mathrm{~mm}$ ) and the overlap with the strong resonances of Ins. Therefore, the measurement of the Gly concentration in $\mathrm{CO}, \mathrm{HI}$ and ST was performed at $\mathrm{TE}=20 \mathrm{~ms}$, where the signal contribution from Ins was minimised as described previously (10).

When assessing the level of Gly from the spectra acquired at $\mathrm{TE}=20 \mathrm{~ms}, \mathrm{~T}_{2}$ relaxation of Gly was assumed to be similar to that of $\mathrm{tCr}$. According to previously published $\mathrm{T}_{2}$ values of metabolites at $9.4 \mathrm{~T}(18), \mathrm{T}_{2}$ of singlets ranged from $113 \mathrm{~ms}(\mathrm{tCr})$ to $202 \mathrm{~ms}$ (NAA). Even when assuming that $T_{2}$ of Gly is close to the longest $T_{2}$, i.e. that of NAA, the Gly concentration in these three regions (i.e. $\mathrm{CO}, \mathrm{ST}$ and $\mathrm{HI}$ ) would be $8 \%$ lower than the values calculated using the assumption of a similar $\mathrm{T}_{2}$ to $\mathrm{tCr}$, which is a quantitatively unimportant difference.

Relative to CO, ST and HI, Gly in MO showed a three-fold higher concentration (Fig. 3a), which is in accordance with the reported Gly regional distribution in rat brain $(20,22)$. The high concentration of Gly in $\mathrm{MO}$ is consistent with its neurotransmitter function in this region. Gly has a dual role as a co-agonist for excitatory glutamatergic neurotransmission, by binding $N$-methyl-D-aspartate receptors, and as an inhibitory neurotransmitter, by activating strychnine-sensitive Gly receptors, which are particularly concentrated in MO (1). Defects in glycinergic neurotransmission may result, for example, in motor disorders, such as that observed in hyperekplexia (25), pain perception (26) or idiopathic generalised epilepsies (27). Thus, the concentration of Gly may eventually become a marker of these pathological conditions.

Among the differences in the neurochemical profile of $\mathrm{MO}$ compared with that of the other regions, some are of particular interest, as they may be related to the function of Gly, e.g. the Tau concentration in MO was particularly low. In the central nervous system, Tau not only has an important osmoregulatory function, but is also a modulator of Gly and GABA receptors (28). As an agonist of glycinergic and GABAergic neurotransmission systems, which are abundant in $\mathrm{MO}$, the Tau concentration is likely to be tightly regulated. Interestingly, the other osmolyte, Ins, was found in $\mathrm{MO}$ at similar concentrations to that in other regions, namely $\mathrm{CO}$ and ST. Another marked characteristic of MO was the low 
(a)

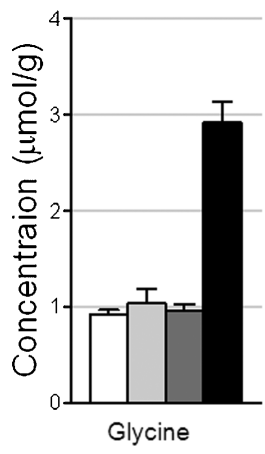

(b)
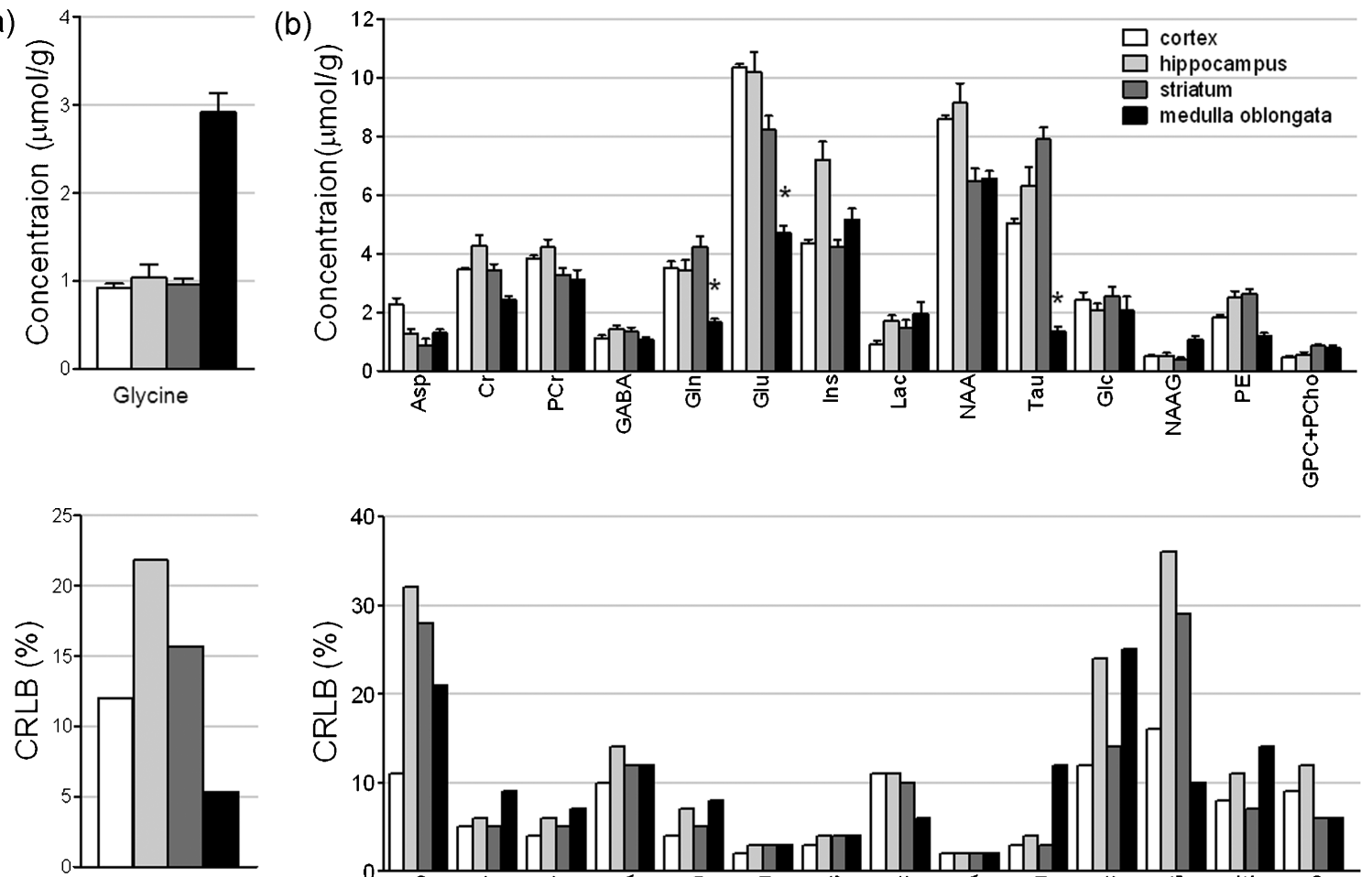

Glycine

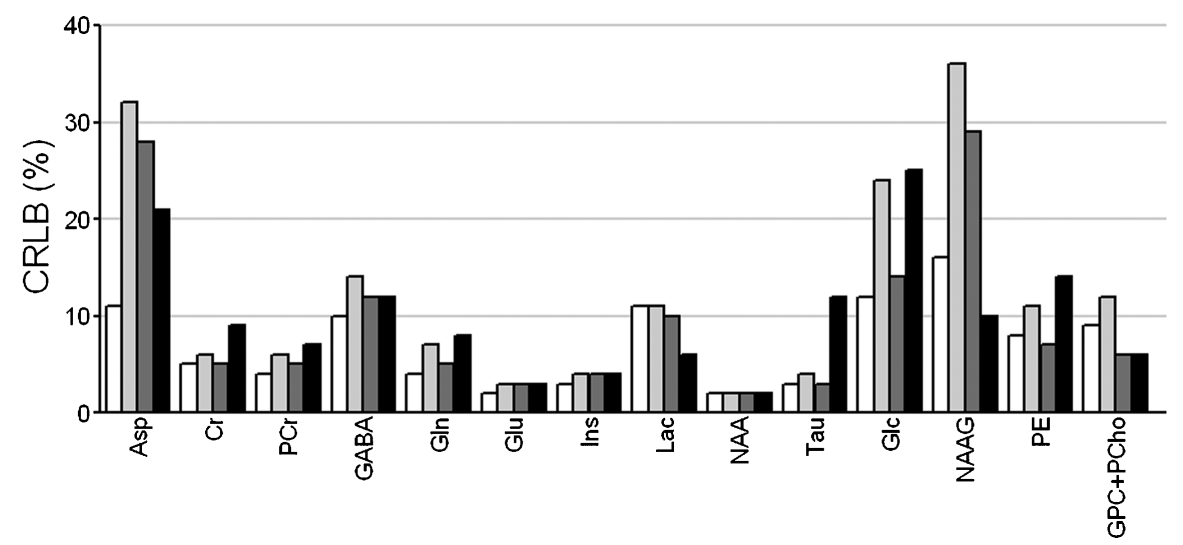

Figure 3. Glycine (a) and other metabolite (b) concentrations and the corresponding Cramér-Rao lower bounds (CRLBs) given by LCModel in the cortex, hippocampus, striatum and medulla oblongata (MO). Data shown as the mean \pm SEM of six different rats. The asterisk indicates the concentrations of metabolites in MO that are significantly different from those in the other three regions $(p<0.001$, two-way ANOVA followed by Bonferroni's multiple comparison test). See text for abbreviations.

concentrations of Glu and Gln when compared with those in other regions (Fig. 3b), being closely related to the prominence of inhibitory systems in $\mathrm{MO}$, and thus perhaps reflecting a lower density of excitatory glutamatergic neurons.

Recently, the volume of MO measured by MRI has been suggested to correlate with the degree of dysfunction in multiple sclerosis, which is a neurodegenerative disorder leading to atrophy of the brain and spinal cord and resulting in motor impairment (5). Hence, the direct monitoring of the neurochemical profile of MO by in vivo ${ }^{1} \mathrm{H}$ NMR spectroscopy may thus provide a complementary investigation of multiple sclerosi$\mathrm{s}$-associated neurodegeneration affecting this brain area.

A few MRS studies, performed in human MO at 1.5 or $3 \mathrm{~T}$ (29-31), have reported exclusively the measurement of three major metabolites, i.e. tCr, NAA and total choline, because of the inferior spectral quality caused by susceptibility effects from the surrounding environment, the low SNR and spectral resolution at low magnetic field strength, and signal loss caused by spectroscopic methods performed at long TE. With an increase in the magnetic field strength, such as at $7 \mathrm{~T}$, the spectral resolution and SNR can be improved, allowing the assessment of a larger number of metabolites in human MO.

\section{CONCLUSION}

We conclude that a neurochemical profile composed of 15 metabolites, including Gly, can be reliably quantified in rat MO by in vivo ${ }^{1} \mathrm{H}$ NMR spectroscopy at $\mathrm{TE}=2.8 \mathrm{~ms}$ at $9.4 \mathrm{~T}$. The ability to measure the neurochemical profile may be useful to investigate MO dysfunction, such as motor impairment in multiple sclerosis. We further conclude that the functional specialisation of distinct brain regions is reflected by specific patterns of metabolite concentrations.

\section{Acknowledgements}

This work was supported by the Centre d'Imagerie BioMédicale (CIBM) of University of Lausanne (UNIL), University of Geneva (UNIGE), Hôpitaux Universitaires de Genève (HUG), Centre Hospitalier Universitaire Vaudois (CHUV) and Ecole Polytechnique Federale de Lausanne (EPFL), and the Leenaards and Jeantet Foundations.

\section{REFERENCES}

1. Betz $\mathrm{H}$, Laube B. Glycine receptors: recent insights into their structural organization and functional diversity. J Neurochem. 2006; 97(6): 1600-1610.

2. Fagundes LD, Rotta LN, Schweigert ID, Valle SC, de Oliveira KR, Kruger AH, Souza KB, Souza DO, Perry MLS. Glycine, serine, and leucine metabolism in different regions of rat central nervous system. Neurochem Res. 2001; 26(3): 245-249.

3. Saransaari P, Oja SS. Mechanisms of glycine release in mouse brain stem slices. Neurochem Res. 2009; 34(2): 286-294. 
4. Colombari E, Sato MA, Cravo SL, Bergamaschi CT, Campos RR Jr, Lopes OU. Role of the medulla oblongata in hypertension. Hypertension. 2001; 38(3 Pt 2): 549-554.

5. Liptak Z, Berger AM, Sampat MP, Charil A, Felsovalyi O, Healy BC, Hildenbrand P, Khoury SJ, Weiner HL, Bakshi R, Guttmann CR. Medulla oblongata volume: a biomarker of spinal cord damage and disability in multiple sclerosis. Am J Neuroradiol. 2008; 29(8): 1465-1470.

6. Pfeuffer J, Tkac I, Provencher SW, Gruetter R. Toward an in vivo neurochemical profile: quantification of 18 metabolites in shortecho-time (1)H NMR spectra of the rat brain. J Magn Reson. 1999; 141(1): 104-120.

7. Tkac I, Rao R, Georgieff MK, Gruetter R. Developmental and regional changes in the neurochemical profile of the rat brain determined by in vivo H-1 NMR spectroscopy. Magn Reson Med. 2003; 50(1): 24-32.

8. Choi C, Bhardwaj PP, Seres P, Kalra S, Tibbo PG, Coupland NJ. Measurement of glycine in human brain by triple refocusing $1 \mathrm{H}-\mathrm{MRS}$ in vivo at 3.0T. Magn Reson Med. 2008; 59(1): 59-64.

9. Gambarota G, Mekle R, Xin L, Hergt M, van der Zwaag W, Krueger G, Gruetter R. In vivo measurement of glycine with short echo-time $1 \mathrm{H}$ MRS in human brain at 7 T. Magma. 2009; 22(1): 1-4.

10. Gambarota G, Xin L, Perazzolo C, Kohler I, Mlynarik V, Gruetter R. In vivo $1 \mathrm{H}$ NMR measurement of glycine in rat brain at $9.4 \mathrm{~T}$ at short echo time. Magn Reson Med. 2008; 60(3): 727-731.

11. Gruetter R, Tkac I. Field mapping without reference scan using asymmetric echo-planar techniques. Magn Reson Med. 2000; 43(2): 319-323.

12. Mlynarik V, Gambarota G, Frenkel H, Gruetter R. Localized shortecho-time proton MR spectroscopy with full signal-intensity acquisition. Magn Reson Med. 2006; 56(5): 965-970.

13. Tkac I, Starcuk Z, Choi IY, Gruetter R. In vivo 1H NMR spectroscopy of rat brain at 1 ms echo time. Magn Reson Med. 1999; 41(4): 649656.

14. Provencher SW. Estimation of metabolite concentrations from localized in vivo proton NMR spectra. Magn Reson Med. 1993; 30(6): 672-679.

15. Mlynarik V, Cudalbu C, Xin L, Gruetter R. 1H NMR spectroscopy of rat brain in vivo at 14.1 Tesla: improvements in quantification of the neurochemical profile. J Magn Reson. 2008; 194(2): 163-168.

16. Snyder J, Thompson RB, Wild JM, Wilman AH. Strongly coupled versus uncoupled spin response to radio frequency interference effects: application to glutamate and glutamine in spectroscopic imaging. NMR Biomed. 2008; 21(4): 402-409.

17. Schwab M, Bauer R, Zwiener U. The distribution of normal brain water content in Wistar rats and its increase due to ischemia. Brain Res. 1997; 749(1): 82-87.

18. Xin L, Gambarota G, Mlynarik V, Gruetter R. Proton T2 relaxation time of J-coupled cerebral metabolites in rat brain at 9.4 T. NMR Biomed. 2008; 21(4): 396-401.
19. Stornetta RL, McQuiston TJ, Guyenet PG. GABAergic and glycinergic presympathetic neurons of rat medulla oblongata identified by retrograde transport of pseudorabies virus and in situ hybridization. J Comp Neurol. 2004; 479(3): 257-270.

20. Suda M, Honma T, Miyagawa M, Wang RS. Alteration of brain levels of neurotransmitters and amino acids in male F344 rats induced by three-week repeated inhalation exposure to 1-bromopropane. Ind Health. 2008; 46(4): 348-359.

21. Aprison MH, Shank RP, Davidoff RA. A comparison of the concentration of glycine, a transmitter suspect, in different areas of the brain and spinal cord in seven different vertebrates. Comp Biochem Physiol. 1969; 28(3): 1345-1355.

22. Cutler RW, Dudzinski DS. Regional changes in amino acid content in developing rat brain. J Neurochem. 1974; 23(5): 1005-1009.

23. Marjanska M, Henry PG, Ugurbil K, Gruetter R. Editing through multiple bonds: threonine detection. Magn Reson Med. 2008; 59(2): 245-251.

24. Choi C, Coupland NJ, Kalra S, Bhardwaj PP, Malykhin N, Allen PS. Proton spectral editing for discrimination of lactate and threonine $1.31 \mathrm{ppm}$ resonances in human brain in vivo. Magn Reson Med. 2006; 56(3): 660-665.

25. Shiang R, Ryan SG, Zhu YZ, Hahn AF, Oconnell P, Wasmuth JJ. Mutations in the alpha-1 subunit of the inhibitory glycine receptor cause the dominant neurologic disorder, hyperekplexia. Nat Genet. 1993; 5(4): 351-358.

26. Ahmadi S, Lippross S, Neuhuber WL, Zeilhofer HU. PGE(2) selectively blocks inhibitory glycinergic neurotransmission onto rat superficial dorsal horn neurons. Nat Neurosci. 2002; 5(1): 34-40.

27. Sobetzko D, Sander T, Becker CM. Genetic variation of the human glycine receptor subunit genes GLRA3 and GLRB and susceptibility to idiopathic generalized epilepsies. Am J Med Genet. 2001; 105(6): 534-538.

28. Albrecht J, Schousboe A. Taurine interaction with neurotransmitter receptors in the CNS: an update. Neurochem Res. 2005; 30(12): 1615-1621.

29. Galanaud D, Le Fur Y, Nicoli F, Denis B, Confort-Gouny S, Ranjeva JP, Viout P, Pelletier J, Cozzone PJ. Regional metabolite levels of the normal posterior fossa studied by proton chemical shift imaging. Magma. 2001; 13(2): 127-133.

30. Mascalchi M, Brugnoli R, Guerrini L, Belli G, Nistri M, Politi LS, Gavazzi C, Lolli F, Argenti G, Villari N. Single-voxel long TE 1H-MR spectroscopy of the normal brainstem and cerebellum. J Magn Reson Imaging. 2002; 16(5): 532-537.

31. Edden RA, Bonekamp D, Smith MA, Dubey P, Barker PB, Proton MR spectroscopic imaging of the medulla and cervical spinal cord. J Magn Reson Imaging. 2007; 26(4): 1101-1105. 